\title{
Basık yörüngeli erken B tipi örten çift sistemlerin eksen dönmesi analizi: V466 Car, V529 Car ve MN Tra
}

\author{
Ahmet BULUT ${ }^{1, *}$, İbrahim BULUT ${ }^{2}$ \\ ${ }^{1}$ Çanakkale Onsekiz Mart Üniversitesi Fen Edebiyat Fakültesi Fizik Bölümü, Terzioğlu Kampüsü, \\ Çanakkale. \\ ${ }^{2}$ Çanakkale Onsekiz Mart Üniversitesi Fen Edebiyat Fakültesi, Uzay Bilimleri ve Teknolojileri Bölümü, \\ Terzioğlu Kampüsü, Çanakkale. \\ Geliş Tarihi (Received Date): 14.11.2018 \\ Kabul Tarihi (Accepted Date): 14.05.2019
}

\section{Özet}

Bu çalışmada, basık yörüngeli örten çift sistemler içinden seçilen V466 Car, V529 Car ve MN Tra sistemlerinin eksen dönmesi analizleri $(O-C)$ diyagramları kullanilarak yapılmış, eksen dönme parametreleri ilk kez hesaplanmıştır. Eksen dönmesi analizi için kullanılan minimum zamanları $(\mathrm{O}-\mathrm{C})$ Gateway veri tabanından alınmıştır. Analizler sonucunda V466 Car, V529 Car ve MN Tra sistemlerinin yörünge diş merkezlikleri, sirasiyla, $0.205 \pm 0.035,0.497 \pm 0.077,0.282 \pm 0.042$, enberi noktasinın boylamındaki ilerlemelerin hızı $0.0194 \pm 0.0013,0.0055 \pm 0.0016,0.0026 \pm 0.0007 \%$ çevrim, bunlara karşıllı gelen eksen dönme dönemleri ise $175 \pm 12,858 \pm 203,894 \pm 204$ yll, olarak elde edilmiştir.

Anahtar kelimeler: Örten çift yıldızlar, eksen dönmesi, V466 Car, V529 Car, MN Tra.

\section{Apsidal motion analysis of early B-type eclipsing binary systems: V466 Car, V529 Car and MN Tra}

\begin{abstract}
\footnotetext{
*Ahmet BULUT, abulut@comu.edu.tr, https://orcid.org/0000-0002-7215-926X

İbrahim BULUT, ibulut@comu.edu.tr, https://orcid.org/0000-0002-7128-8887
}

In the present study, the eclipsing binaries which displaying apsidal motion effect in their orbits, V466 Car, V529 Car and MN Tra were analyzed by using (O-C) diyagrams to obtain the parameters of apsidal motion. The minima times taken from $(\mathrm{O}-\mathrm{C})$ Gateway database were used for the apsidal motion analysis. We found the orbital 
eccentricities $0.205 \pm 0.035,0.497 \pm 0.077$ and $0.282 \pm 0.042$, the rates of change of the longitude of periastron $0.0194 \pm 0.0013,0.0055 \pm 0.0016,0.0026 \pm 0.0007 \%$ cycle, corresponding to the apsidal motion periods of about $175 \pm 12,858 \pm 203$ and $894 \pm$ 204 years, for V466 Car, V529 Car and MN Tra, respectively.

Keywords: Eclipsing binaries, apsidal motion, V466 Car, V529 Car, MN Tra.

\section{Giriş}

Çift yıldız sistemleri, yıldızlara ait çok sayıda fiziksel (kütle, yarıçap, sıcaklık, 1şınım gücü gibi) ve yörünge parametresinin bulunması için önemli sistemlerdir. Basık yörüngeli örten çift yıldızlarda gözlenen ve yörünge asal eksenin uzayda dönmesi olarak tanımlanan eksen dönmesi üzerine yapılan çalışmalar, yıldızların içyapılarına ve evrimine ilişkin önemli sonuçlara ulaşılmasını sağlar [1, 2]. Ayrıca eksen dönmesi çalışmaları görecelik kuramının Güneş Sistemi dışında test edilmesine de olanak sunar. $\mathrm{Bu}$ çalışmaların duyarlılığı, eksen dönmesi parametreleri iyi bilinen çift sistemlerin sayısına bağlıdır.

Bu çalışmada, üç basık yörüngeli örten çift sistemin (V466 Car, V529 Car ve MN Tra) eksen dönmesi analizi ilk kez yapılarak sunulmuştur. Bu sistemler, [3], tarafindan yayınlanan basık yörüngeli örten çift yıldızlar kataloğunda yer almaktadır. Her üç sistem içinde şimdiye kadar hiçbir tayfsal çalışma bulunmamaktadır. Bu çalışma basık yörüngeli yeni örten çift yıldızların eksen dönmesi analizleri üzerine yaptığımız çalışmaların (örneğin bkz. [4]) bir devamıdır.

\section{Eksen dönmesi analizleri}

Basık yörüngeli örten çift yıldız sistemlerinin birinci ve ikinci minimum zamanları ile oluşturulan $(O-C)$ diyagramları zit fazlı iki sinüs eğrisi oluşturur. Sinüs eğrisinin dönemi eksen dönmesi dönemini verir. Çift yıldızlara ilişkin eksen dönme hareketinin dönemleri birkaç yıldan binlerce yılla kadar değişen geniş bir aralıkta olabilir.

$\mathrm{Bu}$ çalışmadaki üç sisteminde eksen dönmesi parametreleri $(O-C)$ diyagramlarının analiziyle hesaplanmıştır. Eksen dönmesi analizi için $[5,6]$, tarafından geliştirilen yöntemi temel alan [7], tarafında yazılan bilgisayar programı kullanılmıştır. $\mathrm{Bu}$ yöntemde dış merkezliğin beşinci dereceden terimlerini içeren [6], tarafından verilen denkleme en küçük kareler yöntemiyle ardışık işlemler (iterasyonlar) yapılarak çözüm gerçekleştirilmektedir. Böylelikle beş temel değişen $\left(T_{0}, P_{s}, e, \omega_{0}, \quad \omega\right)$ hesaplanmaktadır. Bunlardan enberi noktasının boylamı $(\omega)$ aşağıdaki doğrusal ifadeyle tanımlanmaktadır.

$\omega=\omega_{0}+\dot{\omega} E$

Burada $(\omega)$ enberi notasının boylamındaki ilerleme miktarını, $\left(T_{0}\right)$ başlangıç zamanını, $\left(\omega_{0}\right)$ ise başlangıç zamanındaki enberi noktasının boylamını göstermektedir. Yıldızıl dönem $\left(P_{s}\right)$ ile kavuşum (anomalistik) dönemi $\left(P_{a}\right)$ arasındaki ilişki

$P_{s}=P_{a}\left(1-\dot{\omega} / 360^{\circ}\right)$ 
ifadesiyle verilir. Buradan eksen dönmesi dönemi $(U)$ ise aşağıdaki ifadeyle hesaplanır.

$U=360^{\circ} P_{a} / \dot{\omega}$

Eksen dönmesi analizi yapılan 3 sisteme ilişkin minimum zamanları $(O-C)$ Gateway [8], veri tabanından alınmıştır.

\subsection{V466 Car}

V466 Car (GSC 08925-02854, HD 70333, HIP 40666, NSV 4003, PPM 356626, SAO 250181, TYC 8925-2856-1, $\alpha_{2000}=08^{\mathrm{h}} 18^{\mathrm{m}} 5^{\mathrm{s}} .024, \delta_{2000}=-60^{\circ} 18^{\prime} 50^{\prime \prime} .02, V_{\operatorname{mak}}=7^{\mathrm{m}} .29$, $P=3.455887$ gün, tayf türü $\mathrm{B} 8 / 9 \mathrm{~V}$ ) olan sistemin ilk gözlemleri [9], tarafindan yapılmıştır. Değişen olduğu ise Hipparcos uydusu gözlemleriyle belirlenmiştir. Tayf türü [10], tarafından B8/9V olarak vermiştir. Sistemin Hipparcos uydusu [11], gözlemlerinden elde edilen 1şı eğrisi [12], tarafından analiz edilmiştir. Bu analizlerde yan minimumun $\sim 0.37$ evresinde, yörünge diş merkezliğinin ise 0.235 olduğu hesaplanmıştır.

V466 Car'ın eksen dönmesi analizinde, (O-C) Gateway veri tabanından alınan, 11 tanesi birinci minimum olmak üzere, toplam 18 tane fotometrik minimum zamanı kullanılmıştır. Analizlerde yörünge eğimi için [12]'nin verdiği ( $\left.i=81^{\circ} .18\right)$ değer alınmıştır. Analizle sonucu elde edilen eksen dönmesi parametreleri Tablo 1'de, sistemin $(O-C)$ eğrisi ve ona yapılan fit eğrisi Şekil 1'de kuramsal eğriden olan farklar ise Şekil 2'de verilmiştir.

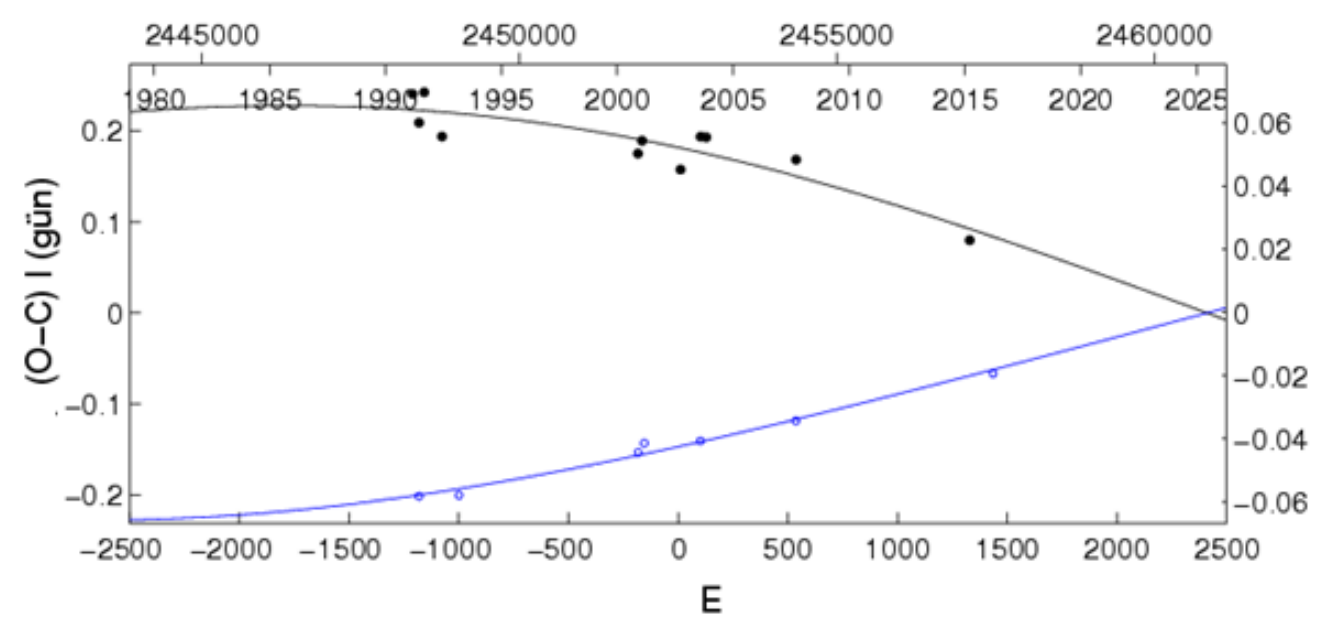

Şekil 1. V466 Car'ın $(O-C)$ diyagramı. Semboller gözlenen $(O-C)$ değerlerini sürekli çizgiler ise kuramsal eğrileri göstermektedir (Sembollerde içi dolu olan I minimum ve boş olan II minimum dairelerle gösterilmiştir). 


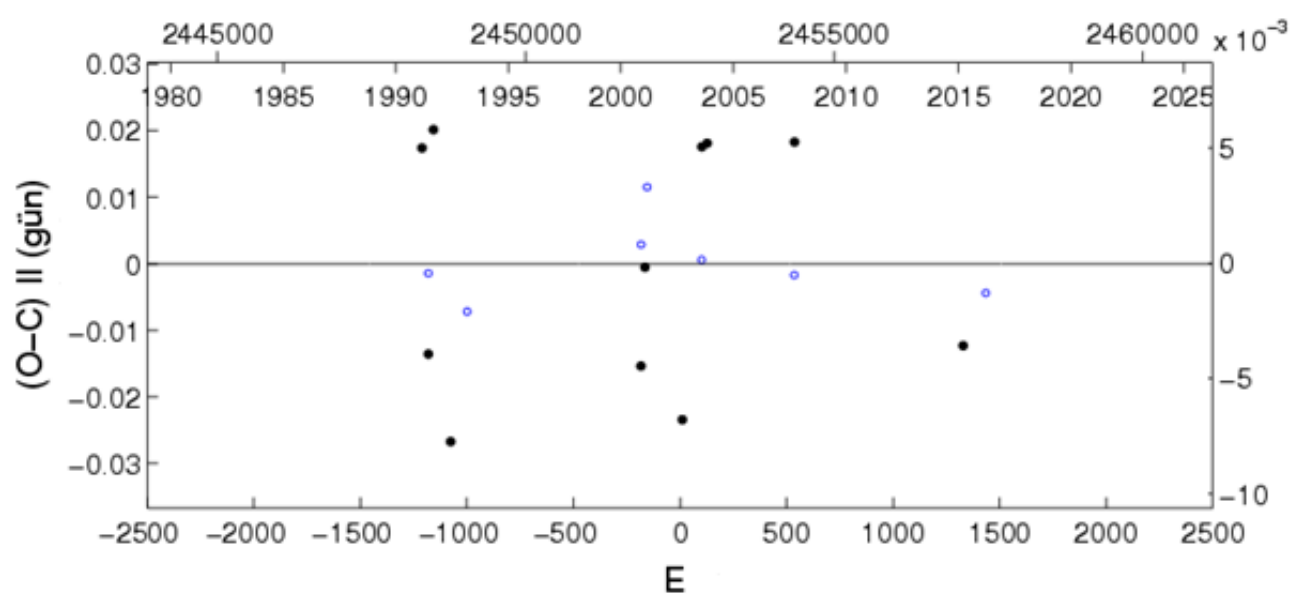

Şekil 2. V466 Car Cas’ın $(O-C)$ değişiminin eksen dönmesi temsilinden olan farklar.

\subsection{V529 Car}

V529 Car (CD-62 492, HD 95993, HIP 54026, 2MASS J11031282-6303598, SAO 251240, TYC 8962-344-1, Gaia DR1 5240908657258193536, $\alpha_{2000}=11^{\mathrm{h}} 03^{\mathrm{m}} 12^{\mathrm{s}} .835$, $\delta_{2000}=-63^{\circ} 03^{\prime} 59^{\prime \prime} .848, V_{\text {mak }}=8^{\mathrm{m}} .18$, tayf türü $=\mathrm{B} 8 \mathrm{~V}$ ve $P=4.74449$ gün) güney yarımkürede yer alan basık yörüngeli bir çift yıldızı sistemidir. V529 Car sisteminin değişen oduğu Hipparcos uydusu tarafından keşfedilmiştir. Çift yıldızın tayf türün B8V olarak vermiştir [10]. Sistemin Hipparcos uydusu gözlemlerinden elde dilen 1şık eğrisi [12], tarafından analiz edilerek fotometrik parametreleri belirlenmiştir. Buna göre yörünge dış merkezliği $(e=0.133)$ ve yörünge eğim açısı $\left(i=79^{\circ} .85\right)$ olarak bulunmuştur.

V529 Car'ın (O-C) Gateway veri tabanında 22 tane fotometrik minimum zamanı yer almaktadır. Bunlardan 12 tanesi birinci minimum, 10 tanesi de ikinci minimumdur. Sistemin eksen dönmesi analizinde bu minimum zamanları kullanılmıştır. Sistemin yörünge eğiminin değeri [12]'den alınmıştır. Analizler sonucunda hesaplanan eksen dönmesi parametreleri Tablo 1'de verilmiştir. Sistemin $(O-C)$ eğrisi ve onun eksen dönmesi ile temsili Şekil 3'de kuramsal eğriden olan farklar ise Şekil 4'de gösterilmiştir. Analizler sistemin yörüngesinin oldukça basık $(e=0.497 \pm 0.077)$ olduğunu göstermektedir.

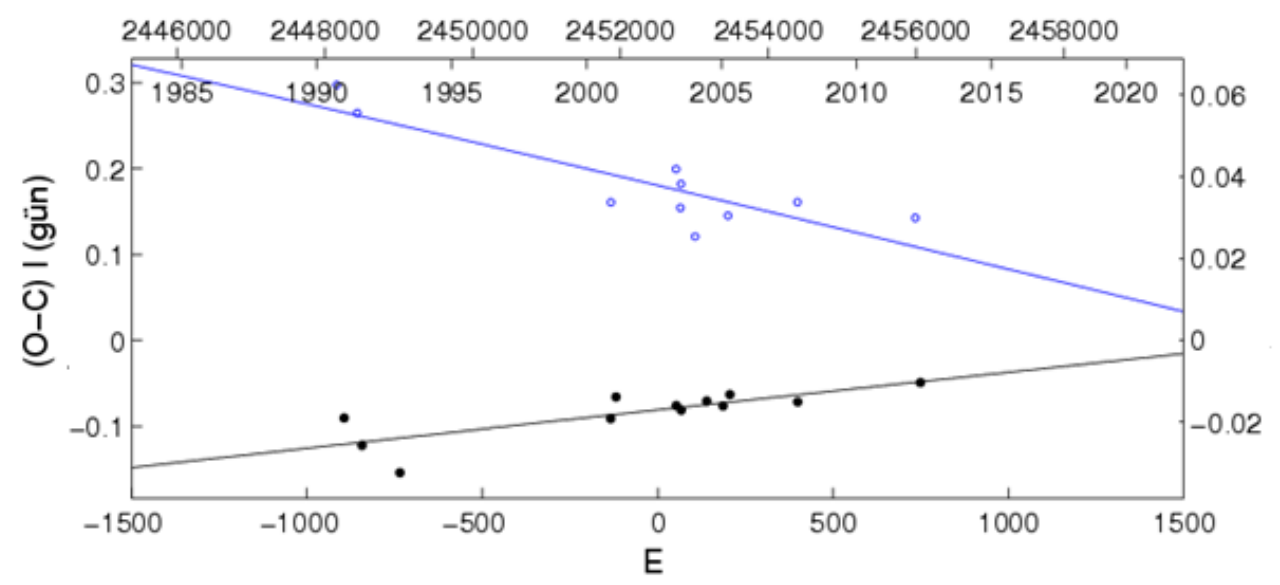

Şekil 3. V529 Car'ın $(O-C)$ değişimi ve bu değişimi temsil eden eğri. 


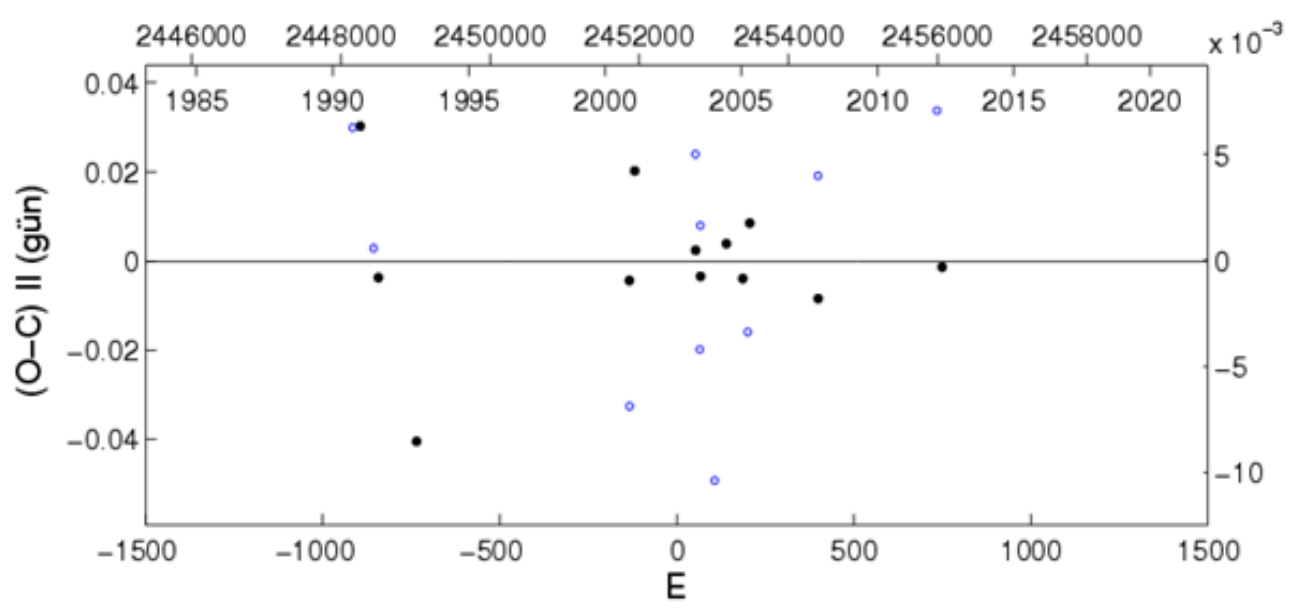

Şekil 4. V529 Car'ın $(O-C)$ değişimininin eksen dönmesi temsilinden olan farklar.

\section{3. $M N$ Tra}

MN Tra (CPD-61 5428, GSC 09027-05249, HIC 78231, HIP 78231, NSV 7349, PPM 361769, SAO 253361, TD1 18670, $\alpha_{2000}=15^{\mathrm{h}} 58^{\mathrm{m}} 28^{\mathrm{s}} .328, \delta_{2000}=-62^{\circ} 03^{\prime} 37^{\prime \prime} .664, V_{\text {mak }}$ $=8^{\mathrm{m}} .55$, tayf türü $=\mathrm{B} 9 \mathrm{~V}$ ve $P=2.379833$ gün) Hipparcos uydusu [13], tarafindan keşfedilen Algol türü bir örten çift yıldız sistemidir. Sistemin Hipparcos gözlemlerinden elde edilen 1 şık eğrisi [12], tarafında analiz edilerek fotometrik parametreler belirlemişlerdir. Bu çalışmada yörünge dış merkezliği $(e=0.081)$ ve yörünge eğim açısı $\left(i=88^{\circ} .84\right)$ derece bulunmuştur.

MN Tra'ın eksen dönmesi analizinde 8 tanesi ikinci minimum olmak üzere, toplam 16 tane fotometrik/CCD minimum zamanı kullanılmıştır. Bu minimum zamanları da $(\mathrm{O}-\mathrm{C})$ Gateway veri tabanından alınmıştır. Sistemin $(O-C)$ eğrisinin ve onun eksen dönmesi ile temsili Şekil 5'de kuramsal eğriden olan farklar ise Şekil 6'de gösterilmiştir. Elde edilen eksen dönmesi parametreleri Tablo 1'te verilmiştir. Yapılan analizlerde sistemin eksen dönme dönemi $894 \pm 204$ yıl, yörünge dışmerkezliği ise $0.282 \pm 0.042$ olarak bulunmuştur. Sonuçlar MN Tra'ın yörüngesinin oldukça bası olduğunu göstermektedir.

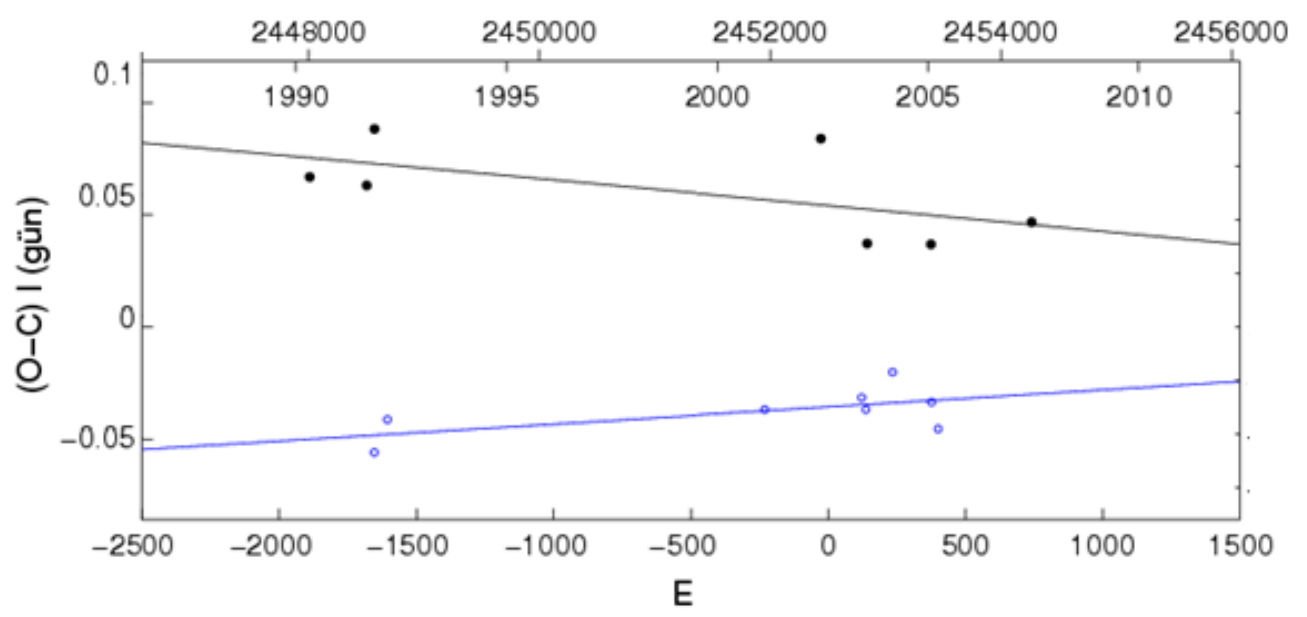

Şekil 5. MN Tra'ın $(O-C)$ değişimi ve bu değişimi eksen dönmesi ile temsil eden eğri. 


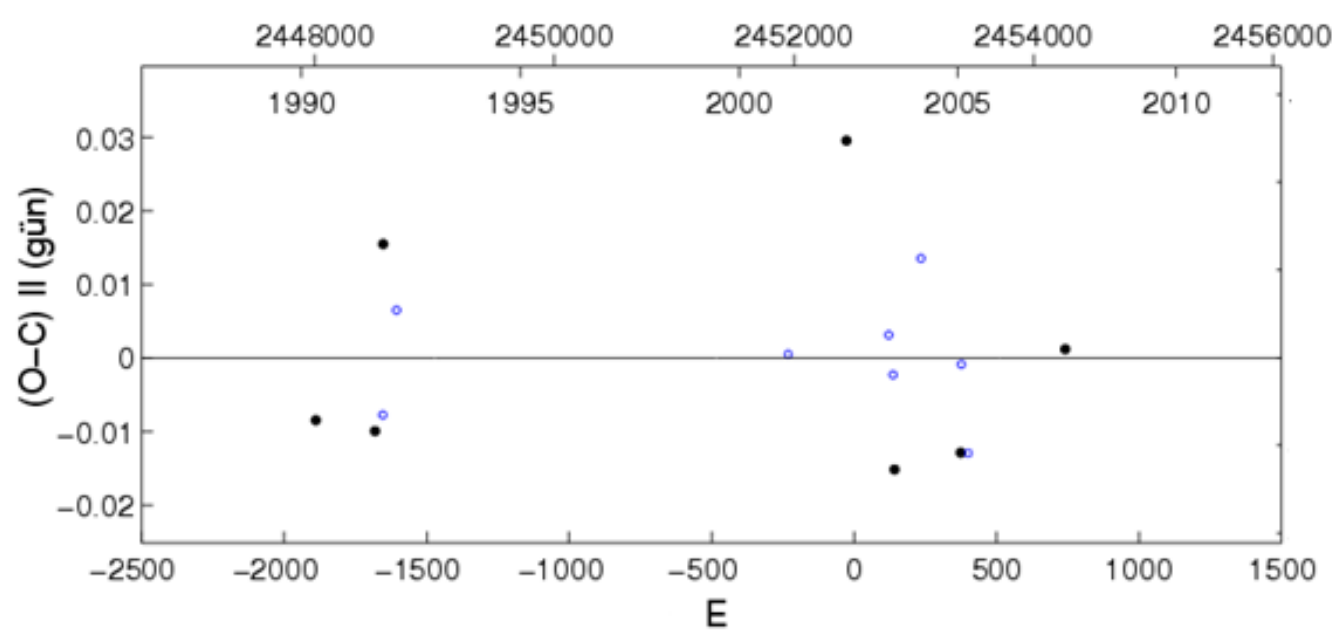

Şekil 6. MN Tra'ın $(O-C)$ verilerinin kuramsal değerlerden olan fark dağılımları.

Tablo 1. V466 Car, V529 Car ve MN Tra’ın yeni ışık elemanları ve eksen dönmesi parametreleri.

\begin{tabular}{lccc}
\hline Parametre & V466 Car & V529 Car & MN Tra \\
\hline$T_{\mathrm{o}}$ (HJD) & $2452501.6814 \pm 0.0452$ & $2452503.3649 \pm 0.0324$ & $2452501.7761 \pm 0.0157$ \\
$P_{s}$ (gün) & $3.455833 \pm 0.000055$ & $4.744583 \pm 0.000066$ & $2.379821 \pm 0.000015$ \\
$P_{a}$ (gün) & $3.4560195 \pm 0.0000547$ & $4.7446543 \pm 0.0000663$ & $2.3798379 \pm 0.0000154$ \\
$e$ & $0.205 \pm 0.035$ & $0.497 \pm 0.077$ & $0.282 \pm 0.042$ \\
$\omega(\%$ çevrim) & $0.0194 \pm 0.0013$ & $0.0055 \pm 0.0016$ & $0.0026 \pm 0.0007$ \\
$\omega_{0}\left({ }^{\circ}\right)$ & $223 \pm 2$ & $80 \pm 2$ & $258 \pm 2$ \\
$U($ yll $)$ & $175 \pm 12$ & $858 \pm 203$ & $894 \pm 204$ \\
\hline
\end{tabular}

\section{Sonuçlar ve tartışma}

Bu çalışmada, [3], kataloğundan eksen dönmesi gösterdiği bildirilen örten çift y1ldız sistemlerinden V466 Car, V529 Car ve MN Tra için eksen dönmesi analizi ilk kez yapılmıştır. Bunun için sistemlerin literatürde mevcut olan minimum zamanları toplanarak, $(O-C)$ eğrileri oluşturulmuş ve analizleri yapılmıştır. Sistemlerin hesaplanan eksen dönmesi parametreleri Tablo 1'de verilmiştir. V466 Car, V529 Car ve MN Tra sistemlerinin eksen dönme dönemleri sirasıyla $175 \pm 12,858 \pm 203$ ve $894 \pm$ 204 y1l olarak bulunmuştur. Bu sonuçlara göre V466 Car'ın eksen dönmesi kısa dönemli diğer sistemlerin ise uzun dönemlidir. Her üç sistemin de yörünge dış merkezliğinin oldukça büyük olduğu görülmektedir.

Çift yıldızlara ilişkin eksen dönme hareketinin dönemleri uzun süreleri kapsamaktadır. $\mathrm{Bu}$ nedenle bu çalışmada analizleri yapılan tüm sistemlerin gelecekte yapılacak yeni minimum zamanı gözlemleri, eksen dönmesi dönemlerinin daha duyarlı biçimde belirlenmesi açısından önemlidir. Yıldızlara ilişkin içyapı sabitlerinin hesaplanması için bileşenlerin mutlak parametrelerinin bilinmesi gereklidir. Gelecekte yapılacak tayfsal çalışmalarla sistemlerin mutlak parametreleri belirlenirse, bileşenlerin gözlemsel içyapı sabiti değerleri bulunup kuramsal yıldız modelleriyle karşılaştırılabilinir. 


\section{Kaynaklar}

[1] Claret, A. ve Giménez, A., Apsidal motion test: confrontation between theory and observations, Inside the stars; Proceedings of the $137^{\text {th }}$ IAU Colloquium, Univ. of Vienna, Austria, Apr. 13-18, 1992. Astronomical Society of the Pacific (ASP Conference Series. Vol. 40), Edited by Werner W. Weiss and Annie Baglin, 469, (1993).

[2] Claret, A. ve Willems, B., New results on the apsidal-motion test to stellar structure and evolution including the effects of dynamic tides, Astronomy and Astrophysics, 388, 518-530 (2002).

[3] Bulut, İ. ve Demircan, O., A new catalogue of eclipsing binary stars with eccentric orbits*, Monthly Notices of the Royal Astronomical Society, 378, 1, 179-181, (2007).

[4] Bulut I. ve Bulut A., BVR Photometric Analysis of GSC 4277-0586 and GSC 3152-1202, Acta Astronomica, 65, 127-138, (2015).

[5] Gimenez, A. ve Garcia-Pelayo, J. M., A new method for the analysis of apsidal motions in eclipsing binaries, Astrophysics and Space Science, 92, 1, 203-222, (1983).

[6] Giménez, A. ve Bastero, M., A Revision of the Ephemeris-Curve Equations for Eclipsing Binaries with Apsidal Motion, Astrophysics and Space Science, 226, 1, 99-107, (1995).

[7] Zasche, P., Liakos, A., Niarchos, P., Wolf, M ve Gazeas, K., Period changes in six contact binaries: WZ And, V803 Aql, DF Hya, PY Lyr, FZ Ori, and AH Tau, New Astronomy, 14, 2, 121-128, (2009).

[8] O-C gateway Database of times of minima (E) and maxima (RR), http://var2.astro.cz/ocgate/, (19.05.2017).

[9] Strohmeier, W., New Southern Variables, Information Bulletin on Variable Stars, 158, 1, (1966).

[10] Houk, N. ve Cowley, A. P., University of Michigan Catalogue of twodimensional spectral types for the HD stars. Volume I. Declinations $-90_{-}$to 53 f0., University of Michigan Catalogue of two-dimensional spectral types for the HD stars. Volume I. Declinations -90_ to -53_f0., by Houk, N.; Cowley, A. P.. Ann Arbor, MI (USA): Department of Astronomy, University of Michigan, $19+452,(1975)$.

[11] ESA, The HIPPARCOS and TYCHO catalogues. Astrometric and photometric star catalogues derived from the ESA HIPPARCOS Space Astrometry Mission, The Hipparcos and Tycho catalogues. Astrometric and photometric star catalogues derived from the ESA Hipparcos Space Astrometry Mission, Publisher: Noordwijk, Netherlands: ESA Publications Division, 1997, Series: ESA SP Series, 1200, (1997).

[12] Bulut, İ. ve Demircan, O., Light curve analyses of new eclipsing binary systems with eccentric orbits, New Astronomy, 13, 4, 252-254, (2008).

[13] Perryman, M. A. C., Lindegren, L., Kovalevsky, J., Hoeg, E., Bastian, U., Bernacca, P. L., Crézé, M., Donati, F., Grenon, M., Grewing, M., van Leeuwen, F., van der Marel, H., Mignard, F., Murray, C. A., Le Poole, R. S., Schrijver, H., Turon, C., Arenou, F., Froeschlé, M. ve Petersen, C. S., The HIPPARCOS Catalogue, Astronomy and Astrophysics, 323, L49-L52, (1997). 